\title{
Tip-sample interactions: Extraction of single molecular pair potentials from force curves
}

\author{
Marc A. Unger, Stephen D. O'Connor, and John D. Baldeschwieler ${ }^{\mathrm{a})}$ \\ Division of Chemistry 127-72, California Institute of Technology, Pasadena, California 91125
}

(Received 24 July 1995; accepted 19 December 1995)

\begin{abstract}
This article describes a method for extracting the true tip-sample potential from an experimental force curve in atomic force microscopy. This potential is not the negative integral of the force curve. Rather, the potential is a more complicated function of the force curve and cantilever spring constant. If information about the shape of the tip is known, a decorrelation may be performed to extract molecular pair potentials from the total tip-sample potential. Applications and limitations of this method are discussed. (C) 1996 American Vacuum Society.
\end{abstract}

\section{INTRODUCTION}

Atomic force microscopy (AFM) is capable of imaging surfaces with high resolution in a noninvasive manner. ${ }^{1-3}$ To perform an AFM experiment, a flexible cantilever with a small protruding tip is brought into contact with a surface; as the tip is scanned, the interaction forces between the electron clouds of the atoms of the tip and the sample perturb the cantilever from its equilibrium position.

In addition to its imaging capability, AFM is also capable of measuring the force between the tip and sample as a function of tip-sample separation, which is analogous to Israchevilli's surface force experiments with a smaller contact area. ${ }^{4,5}$

Several models have been proposed to explain AFM force curves. ${ }^{6-8}$ These models show poor agreement with experimental data. Often, the force curve is assumed to be related to the interaction potential by

$$
F_{\text {meas }}(r)=-\frac{d}{d r} V(r)
$$

where $r$ is the tip-sample separation and $V(r)$ is the potential. However, an experimental force curve does not record the force on the tip as a function of tip-sample distance. Rather, it monitors the force on the tip versus the separation of the cantilever base and the sample. Equation (1) is only correct when the cantilever base and the tip do not move with respect to each other, which is true only in the limit of a very large cantilever spring constant. This error is responsible for the inaccuracy of the negative integral method of reconstructing the tip-sample potential from the force curve.

The chemical nature of the tip and sample must also be considered when interpreting force curves. The potential between tip and sample will be primarily due to the interactions of the surface atoms. ${ }^{9}$ Controlled experiments confirm that the surface chemistry dominates the shape of the force curves. ${ }^{9-11}$

In this article, a thorough analysis of the AFM sample-tip cantilever system will be presented. An accurate expression for the relationship between the force curve and the actual

a) To whom correspondence should be addressed. tip-sample potential will be derived. These results will be compared to the standard method of force curve interpretation by numerical simulation.

A chemical model of the tip-sample interaction will also be developed. This model analyzes the relation between the tip shape, intermolecular potential, and the total potential between tip and sample. Numerical and analytical methods for extracting single intermolecular pair potentials from the total tip-sample potential will be derived, and the limitations of applying these methods to real experimental data will be discussed.

\section{EXTRACTION OF ACCURATE TIP-SAMPLE POTENTIALS FROM FORCE CURVES}

In this section, an accurate analytical expression for the relationship between the force curve and the actual tipsample potential will be derived. This expression will be compared with the standard method of force curve interpretation, and its application to real-world data will be discussed.

\section{A. Theoretical model (analytical)}

The sample-tip-cantilever system will be modeled by replacing the cantilever with a spring of spring constant $k$. In Fig. 1(b), the cantilever deflection $\beta$ is $z-D-c_{\text {eq }}$, where $c_{\text {eq }}$ is the equilibrium length of the spring.

To perform a force curve, the cantilever deflection is monitored as the base is withdrawn from the sample; the distance between the cantilever base and the sample (which is $z$ ) is being controlled, not the distance between the tip and the sample (which is $D$ ). Since a force curve does not accurately describe the force versus distance behavior of the tip versus the sample, we will use the terminology "deflection versus distance curve" [which is denoted DVD curve or $\beta(z)]$.

To find the relationship between $D$ and $z$, an equation for force balance is used:

$$
k\left(z-D-c_{\mathrm{eq}}\right)+-\frac{d}{d D} V_{\mathrm{ts}}(D)=0 .
$$

The first term is the force from the cantilever spring; the second term is the force due to the tip-sample potential. 


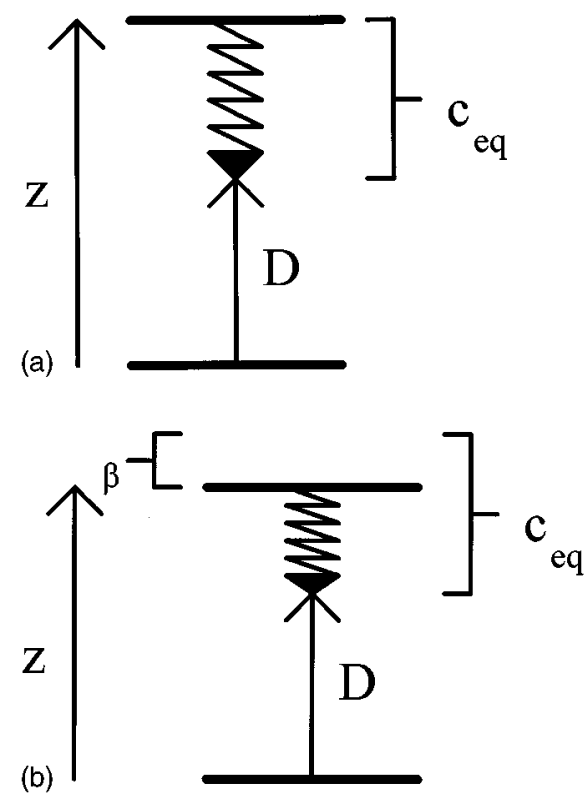

FIG. 1. The tip-sample-cantilever system. The variables $z, D, c_{\text {eq }}$, and $\beta$ are the cantilever-base-sample distance, the tip-sample distance, the equilibrium (undeflected) spring length, and the cantilever deflection, respectively. (a) shows the undeflected cantilever; (b) shows the cantilever deflected by repulsive interaction with the surface.

When the cantilever is in contact (a compressed state), the cantilever force is negative. The compressed position is in a repulsive regime of the tip-sample potential, therefore the negative derivative will be positive. The total force will be zero at equilibrium.

As the cantilever base is moved, the change in the tip sample distance $(d D / d z)$ is

$$
\frac{d D}{d z}=\frac{k}{k+\left(d^{2} / d D^{2}\right) V_{\mathrm{ts}}(D)} .
$$

For the limiting case where $k \gg d^{2} V_{\mathrm{ts}} / \mathrm{dD}^{2}$, the cantilever is not bent by the tip-sample potential, and $D$ changes exactly the same amount as $z$. For $k \ll d^{2} V_{\mathrm{ts}} / \mathrm{dD}^{2}$, the tip-sample potential is very stiff and the tip position remains fixed relative to the sample regardless of how the cantilever base is moved.

Experimentally, a force curve measures the cantilever deflection $(\beta)$ as a function of $z$. Therefore,

$$
\begin{aligned}
\beta^{\prime}(z)=\frac{d \beta(z)}{d z} & =\frac{d}{d z}\left(z-D-c_{\mathrm{eq}}\right) \\
& =1-\frac{d D}{d z} \\
& =\frac{\left(d^{2} / d D^{2}\right) V_{\mathrm{ts}}(D)}{k+\left(d^{2} / d D^{2}\right) V_{\mathrm{ts}}(D)},
\end{aligned}
$$

which can be rearranged to give

$$
V_{\mathrm{ts}}(D)=\iint \frac{k\left[\beta^{\prime}(z)\right]}{\left[1-\beta^{\prime}(z)\right]} d D d D
$$

In order to perform this integral, Eq. (5) must be written as a function of $D$, not $z$ :

$$
\beta^{\prime}(z)=\beta^{\prime}[D(z)]
$$

and from Eq. (4):

$$
D(z)=\int 1-\beta^{\prime}(z) d z .
$$

Therefore, in order to convert from a DVD curve to the actual potential, $D(z)$ must be computed from $\beta(z)$ using Eq. (7). Then, $V_{\mathrm{ts}}(\mathrm{D})$ can be determined using Eq. (5).

\section{B. Numerical simulation}

Computer code has been developed to convert the DVD curve to the tip-sample potential. Figure 2 presents a pseudo "hard-wall" potential. The DVD curve that would be experimentally measured due to this potential was calculated; from this DVD curve, the potential was "reconstructed" by both the negative integral method and the method outlined above. The zero point on the numerical integration was determined by assuming that the force on the cantilever is zero when the tip-sample separation is large. Figure 3 is the same simulation for a Morse potential.

The simulations show that there can be a "loopback" in the calculated DVD curve. Examination of the analytical expressions in Sec. II A shows that this loopback can only occur when the tip-sample potential is attractive and has a second derivative greater than the spring constant of the cantilever that is, when the "stiffness" of the attractive force momentarily exceeds the stiffness of the cantilever. This manifestation generates the "jump to contact" and "snapoff" commonly observed in experimental DVD curves.

The loopback observed in Fig. 3(B) is not simply a mathematical artifact. The mathematics used in this article produce one DVD curve that is a function of the potential and cantilever spring constant. The loopback in the curve implies that for some values of $z$ there are two positions at which the cantilever exibits a local energy minimum. While approaching the sample, the cantilever would remain in the same potential well until it reaches the point of discontinuity where the DVD curve starts to loop back. At this point, the cantilever would jump to the other local minimum (jump to contact). When retracting the tip, the point of discontinuity is further away from the sample, as shown in Fig. 3(b). This explanation also predicts that the snap-off point will always be further from the sample than the jump to contact.

Note that in the case where there is a jump to contact and snap-off, the loopback region of the DVD curve cannot be measured. This gap limits the utility of the method discussed above for reconstructing tip-sample potentials. The problem may be minimized by using a cantilever with a high spring constant. A numerical algorithm that is insensitive to this "loopback" region is in development. ${ }^{15}$

These simulations also show that the DVD-to-potential transformation is highly sensitive to noise. Application of a smoothing algorithm to the DVD curve prior to the conversion corrects this problem. The smoothing algorithm em- 


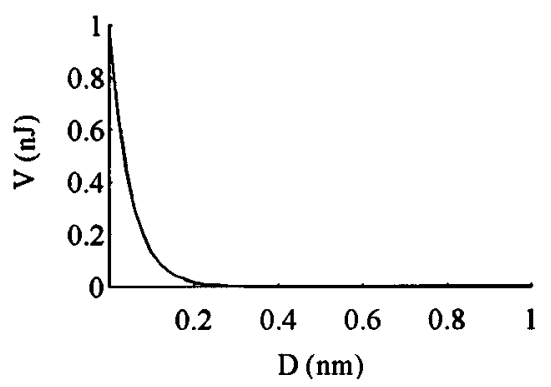

A. Pseudo hard-wall potential

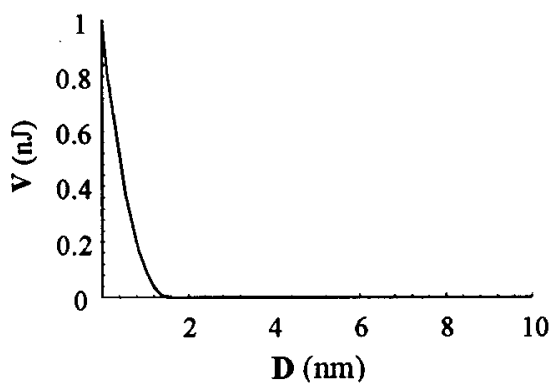

C. Potential from Negative Integral Method

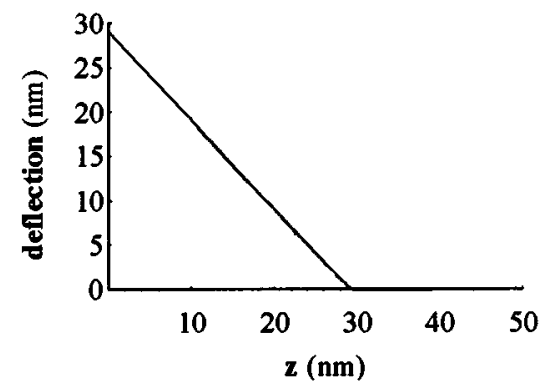

B. Calculated DVD curve

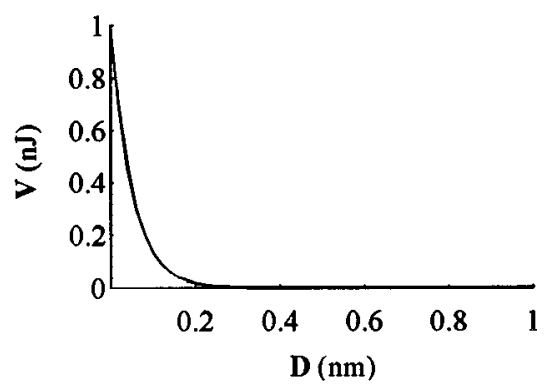

D. Potential from method described here

FIG. 2. Pseudohard wall potential simulation. The cantilever spring constant $=1 \mathrm{~N} / \mathrm{m}$. All the DVD curves have been multiplied by -1 to show deflection away from the surface as positive. The potential derived with the negative integral method has been truncated at $1 \mathrm{~nJ}$ for comparison. Notice the difference in length scale.

ployed was a simple, three-point moving window: the new value at each point is the average of the point and its two nearest neighbors. For $0.1 \mathrm{~nm}$ of noise and a cantilever with a $0.1 \mathrm{~N} / \mathrm{m}$ spring constant, five passes of this smoothing algorithm were usually sufficient. For $0.25 \mathrm{~nm}$ of noise, ten passes were sufficient. The smoothing was considered complete when the conversion results were reproducible.

Two other factors may contribute to the inaccuracy of experimental force curves: scanner calibration and spring constant uncertainty. For most commercially available systems, scanners are calibrated by the manufacturer. However, hysteresis and repoling may alter the calibration accuracy. It is preferable to monitor the sample position simultaneously with an alternative mechanism (such as interferometry or capacitance) while the force curve is being performed. Also, Eq. (5) is dependent upon the cantilever spring constant. Typical manufacturer specifications can be very inaccurate and imprecise. ${ }^{12}$ A number of highly accurate methods have been developed to determine the cantilever spring constant experimentally. $^{12-14}$

\section{EXTRACTION OF INTERMOLECULAR PAIR POTENTIALS FROM TIP-SAMPLE POTENTIALS}

In this section, the relationship between intermolecular pair potentials, tip geometry, and total tip-sample potential will be analyzed. The tip-sample potential can be modeled as the potential between rigidly bound head groups on the tip and sample, i.e., $V_{\mathrm{ts}}(\mathrm{D})=\mathrm{V}_{\mathrm{mol}}(D)$. Finally, the tip-sample interaction will be represented by the summation of all the pairwise interactions of chemical "head groups." It will be shown that the tip-sample potential is a cross correlation of the intermolecular potential with a function of the tip geometry. Extraction of the intermolecular potential (or information about the shape of the tip) from the tip-sample potential is possible by either direct decorrelation or an analytical fitting method; analytical fitting will be shown to be the more useful of the two for real data.

\section{A. Sum $\rightarrow$ Correlation}

The total tip-sample potential can be written as a sum of the single potentials at variable distances $(\alpha)$ (see Fig. 4):

$$
\begin{aligned}
V_{\mathrm{ts}}(D)= & V_{\mathrm{mol}}\left(D+\alpha_{1}\right)+V_{\mathrm{mol}}\left(D+\alpha_{2}\right)+V_{\mathrm{mol}}\left(D+\alpha_{3}\right) \\
& +\cdots ;
\end{aligned}
$$

and for $n$ groups:

$$
V_{\mathrm{ts}}(D)=\sum_{i=1}^{n} V_{\mathrm{mol}}\left(D+\alpha_{i}\right) .
$$

As the limit of a continuum of $\alpha_{i}$ 's is approached:

$$
V_{\mathrm{ts}}(D)=\int_{-\infty}^{\infty} V_{\mathrm{mol}}(D+\alpha) \rho(\alpha) d \alpha,
$$




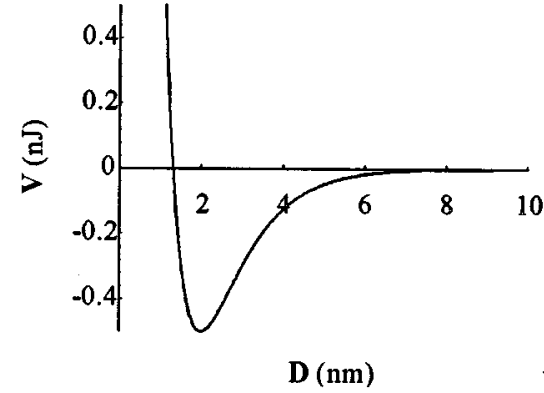

A. Morse potential

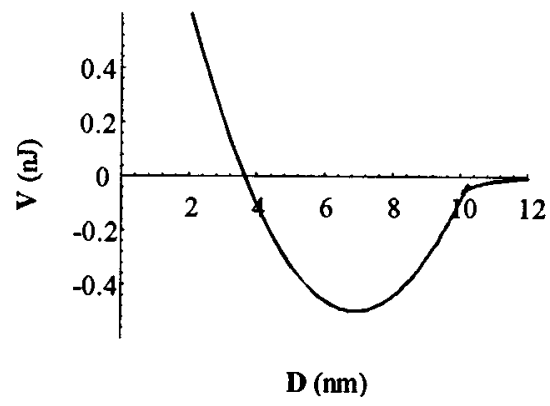

C. Potential from Negative integral method

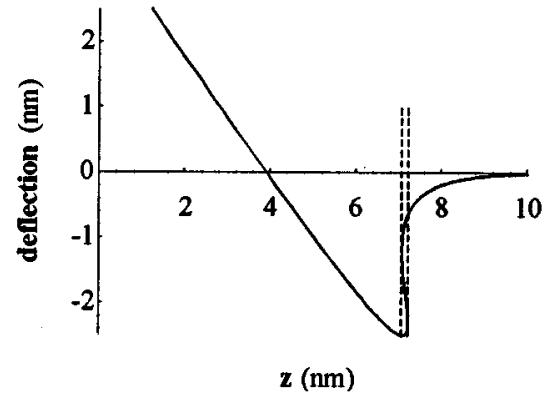

B. Calculated DVD curve

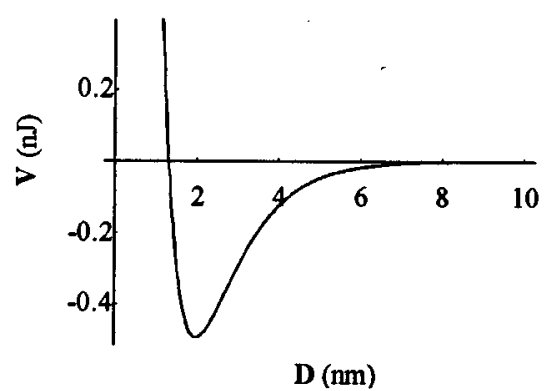

D. Potential from method described here

FIG. 3. Morse potential simulation. The cantilever spring constant $=0.1 \mathrm{~N} / \mathrm{m}$. The distance origin is arbitrary. Note the loopback in the DVD curve. The dotted lines denote the points of instability that manifest themselves in the jump to contact and snap-off commonly seen in force curves.

where $\rho$ is the distribution of distances. This integral is a cross correlation of $V_{\text {mol }}(D)$ and $\rho(D)$, a close mathematical relative of the convolution:

$f(x)^{*} g(x)=\int_{-\infty}^{\infty} f(x-\alpha) g(\alpha) d \alpha \quad$ (convolution),

$f(x) \otimes g(x)=\int_{-\infty}^{\infty} f(x+\alpha) g(\alpha) d \alpha \quad($ cross correlation)

$V_{\mathrm{mol}}(D) \otimes \rho(D)=\int_{-\infty}^{\infty} V_{\mathrm{mol}}(D+\alpha) \rho(\alpha) d \alpha$

(tip-sample cross correlation).

Therefore,

$$
V_{\mathrm{ts}}(D)=V_{\text {mol }}(D) \otimes \rho(D) .
$$

This mathematical model is useful since (i) it allows us to find the tip-sample potential easily from the intermolecular potential and the shape of the tip; (ii) the correlation may be found by a Fourier transform method if the integral cannot be performed analytically; (iii) the Fourier transform method can be used to perform a decorrelation, allowing $V_{\mathrm{mol}}$ to be determined if $\rho$ and $V_{\text {ts }}$ are known.

Application of the definition of the Fourier transform to the convolution integral gives

$$
\begin{aligned}
& f(x)^{*} g(x)=F^{-1}\{F(f(x)) \cdot F[g(x)]\} \quad \text { (convolution), } \\
& f(x) \otimes g(x)=F^{-1}[F[f(x)] \cdot F[g(-x)]] \quad \text { (correlation), } \\
& V_{\mathrm{mol}}(D) \otimes \rho(D)=F^{-1}\left\{F\left[V_{\mathrm{mol}}(D)\right] \cdot F[\rho(-D)]\right\}
\end{aligned}
$$$$
\text { (tip-sample correlation). }
$$

$F$ and $F^{-1}$ represent the Fourier transform and inverse Fourier transform, respectively.

\section{B. Direct decorrelation}

\section{Intermolecular pair potential $V_{\text {mol }}(D)$}

Straightforward manipulation of the correlation equations (14) and (17) gives

$$
V_{\mathrm{mol}}(D)=F^{-1}\left(\frac{F\left[V_{\mathrm{ts}}(D)\right]}{F[\rho(-D)]}\right) .
$$

If $V_{\mathrm{ts}}(\mathrm{D})$ and $\rho(D)$ are known, it is in principle possible to extract the intermolecular pair potential of the head groups $\left[V_{\mathrm{mol}}(D)\right]$.

\section{Distance distribution $\rho$}

The correlation equations (14) and (17) can also be rearranged to give 


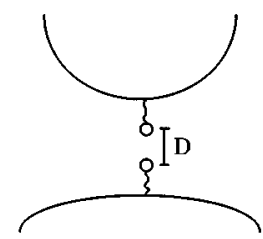

Lone groups

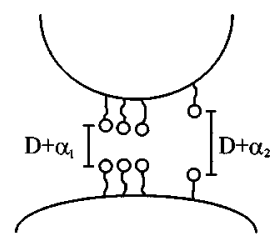

Multiple groups
FIG. 4. Single and multiple head groups. $D$ and $D+\alpha_{n}$ are the intermolecular distances for a single pair (a) and an ensemble (b) of molecular head groups.

$$
\rho(-D)=F^{-1}\left(\frac{F\left[V_{\mathrm{ts}}(D)\right]}{F\left[V_{\mathrm{mol}}(D)\right]}\right) .
$$

Therefore, if $V_{\mathrm{ts}}(\mathrm{D})$ and $V_{\mathrm{mol}}(D)$ are known, it is in principle possible to extract $\rho(D)$, the head-group distribution function. Note: $\rho(D)$ does not uniquely determine the actual geometry. However, the distribution itself is a valuable piece of information because it allows the radius of curvature at the apex of the probe to be estimated. A distribution that has a large value close to $D=0$ must have a large radius of curvature; a distribution that is near zero at $D=0$ has a small radius of curvature.

\section{Limitations of direct decorrelation}

The decorrelation Eqs. (18) and (19) are analytical expressions; they make the assumption that $V_{\mathrm{ts}}(\mathrm{D})$ is known for all values of $D$. In practice, instrumental limitations restrict the measurement to a finite segment of the DVD curve and thus a finite segment of the potential. Effectively, $V_{\mathrm{ts}}(\mathrm{D})$ is being multiplied by a window function $[w(D)] ; w(D)=1$, where $V_{\mathrm{ts}}(\mathrm{D})$ is known; $w(D)=0$ elsewhere. Therefore, in the Fourier domain, $F\left[V_{\mathrm{ts}}(\mathrm{D})\right]$ must be replaced with $F\left[V_{\mathrm{ts}}(\mathrm{D})\right]^{*} F[w(D)]$ [Eqs. (17) and (18)]. This limitation makes the numerical decorrelation possible only under certain conditions.

If $V_{\mathrm{ts}}(\mathrm{D})$ is zero outside the window, then $w(D) V_{\mathrm{ts}}(\mathrm{D})=\mathrm{V}_{\mathrm{ts}}(\mathrm{D})$, and the information outside the window does not corrupt the data. For example, at the right cutoff (i.e., large $D), \quad V_{\mathrm{ts}}(\mathrm{D})$ approaches zero, and $w(D) V_{\mathrm{ts}}(\mathrm{D}) \approx \mathrm{V}_{\mathrm{ts}}(\mathrm{D})$. However, most potentials become strongly repulsive as $D$ approaches zero. The left cutoff will be in the repulsive regime, where $V_{\mathrm{ts}}(\mathrm{D})$ is large outside the envelope, and the leftmost decorrelated data will be inaccurate up to the width of $\rho(D)$. Therefore, this method is limited to cases where the potential is wider than the correlating function. In practice, a narrow $\rho(D)$ is required, such as planar, tilted square (with a small angle), narrow Gaussians, etc.

\section{Analytical fitting}

A more flexible method of performing decorrelations is to fit experimental data to analytical correlations. This method avoids envelope-limited potentials and is less sensitive to noise than the decorrelation method.

\section{Intermolecular pair potential $V_{\text {mol }}(D)$}

A functional form $V_{\text {mol,ff }}(D)$ must first be chosen to represent the intermolecular potential. The analytical correlation $V_{\text {mol,ff }}(\mathrm{D}) \otimes \rho(\mathrm{D})=\mathrm{V}_{\mathrm{ts}, \mathrm{ff}}$ is carried out, and the experimentally determined $V_{\mathrm{ts}}(\mathrm{D})$ is then fit to $V_{\mathrm{ts}, \mathrm{ff}}$. For example, if a Morse potential is chosen for the functional form of the intermolecular potential and a sphere as our model for the tip shape,

$$
\begin{gathered}
V_{\mathrm{mol}, \mathrm{ff}}(D)=D_{e}\left(\left(1-e^{-\beta\left(D-r_{0}^{4}\right)}\right)^{2}-1 \quad\right. \text { and } \\
\rho(D)=\pi d
\end{gathered}
$$

$\left(D_{e}, \beta\right.$, and $r_{0}$ are the dissociation energy, stiffness, and equilibrium distance of the Morse potential, respectively; $d$ is the diameter of the sphere). The experimentally determined $V_{\mathrm{ts}}(\mathrm{D})$ is then fit to $V_{\mathrm{ts}, \mathrm{ff}}$, where $V_{\mathrm{ts}, \mathrm{ff}}$ is given by

$$
\begin{aligned}
V_{\mathrm{ts}, \mathrm{ff}}(D) & =V_{\mathrm{mol}, \mathrm{ff}}(D) \otimes \rho(D) \\
& =\pi d \frac{D_{e}}{2 \beta} e^{\beta\left(r_{0}-D\right)}\left(e^{\beta\left(r_{0}-D\right)}-4\right) .
\end{aligned}
$$

The fitted parameters $D_{e}, \beta$, and $r_{0}$ then describe our best-fit Morse potential for $V_{\mathrm{mol}}(D)$.

\section{Distance distribution $\rho$}

The use of analytical fitting to find $\rho(D)$ simply reverses the roles of $\rho(D)$ and $V_{\text {mol }}$ in the above procedure. A functional form $\rho_{\mathrm{ff}}(\mathrm{D})$ must first be chosen to represent the geometry of the tip. The analytical correlation $V_{\text {mol }}(D) \otimes \rho_{\mathrm{ff}}(\mathrm{D})=\mathrm{V}_{\mathrm{ts}, \mathrm{ff}}$ is carried out, and the experimentally determined $V_{\mathrm{ts}}(\mathrm{D})$ is then fit to $V_{\mathrm{ts}, \mathrm{ff}}$, as described above. If a sphere $[\rho(D)=\pi d]$ is chosen as our functional form for $\rho(D)$, and a Morse potential as our model for the intermolecular potential, then $V_{\mathrm{ts}, \mathrm{ff}}(\mathrm{D})$ will again be given by Eq. 21 . This time, however, our only fitted parameter is $d$, the radius of the sphere representing the tip.

\section{Limitations}

The primary limitation of the analytical fitting method of decorrelation is the difficulty of carrying out some of the analytical correlations. Analytical correlations for a wide variety of intermolecular potential functional forms and $\rho$ 's have been carried out. ${ }^{15}$

There are several intrinsic limitations that apply to all decorrelation methods. Since $V_{\mathrm{ts}}(\mathrm{D})$ is averaged over $x$ and $y$, the extracted $V_{\text {mol }}(D)$ is also averaged over $x$ and $y$. A smooth $\rho(D)$ is a good approximation only for a reasonably large number of head groups ( $n>100$ or more).

The correlation model makes the assumption that all interactions are pairwise. No account is made for cooperative interactions between neighboring head groups. If necessary, an appropriately weighted $\rho(D)$ could be chosen to include these cooperative interactions before decorrelating.

\section{CONCLUSION}

In this article, an accurate method for deriving tip-sample potentials from AFM force curves has been developed and 
shown to be practical for real-world data. Also, a mathematical technique for determining single molecular pair potentials from these tip-sample potentials has been discussed. In practice, these algorithms may be used by an experimental microscopist in two manners.

First, if the surface chemistry of the AFM tip is carefully controlled, it should be possible to determine an accurate $\rho(D)$ from the measured force curve, since the molecular pair potentials can be estimated. As noted above, many complicated tip geometries may possess similar $\rho(D)$. However, for typical AFM tips, it is possible to accurately estimate the radius of curvature of the apex with this method.

Second, an accurate determination of the tip apex shape will produce an accurate $\rho(D)$. Therefore, if a chemical moiety with an unknown pair potential is attached to the tip, the pair potential can be extracted from the force curve.

${ }^{1}$ G. Binnig, C. F. Quate, and Ch. Gerber, Phys. Rev. Lett. 56, 930 (1986).
${ }^{2}$ T. R. Albrecht and C. F. Quate, J. Appl. Phys. 62, 2599 (1987).

${ }^{3}$ P. K. Hansma, J. P. Quate, and M. Radmacher, Appl. Phys. Lett. 64, 1738 (1994).

${ }^{4}$ N. A. Burnham, R. F. Colton, and H. M. Pollock, Nanotechnology, 4, 64 (1993).

${ }^{5}$ J. N. Israelachivili, Intermolecular and Surface Forces (Academic, New York, 1992), p. 169.

${ }^{6}$ U. Hartmann, J. Vac. Sci. Technol. B 9, 465 (1991).

${ }^{7}$ N. A. Burnham, R. J. Colton, and H. M. Pollock, Phys. Rev. Lett. 69, 144 (1992).

${ }^{8}$ E. Kotomin, A. Shluger, M. Causa, R. Dovesi, and F. Ricca, Surf. Sci. 232, 399 (1990).

${ }^{9}$ C. D. Frisbie, L. F. Rozsnyai, A. Noy, M. J. Wrighton, and C. M. Lieber, Science 265, 2071 (1994).

${ }^{10}$ G. U. Lee, L. Chrisey, and R. K. Colton, Science 266, 771 (1994).

${ }^{11}$ G. U. Lee, D. A. Kidwell, and R. K. Colton, Langmuir 10, 354 (1994).

${ }^{12}$ T. J. Senden and W. A. Ducker, Langmuir 10, 1003 (1994).

${ }^{13}$ J. P. Cleveland, S. Manne, D. Bocek, and P. K. Hansma, Rev. Sci. Instrum. 64, 403 (1993).

${ }^{14}$ J. L. Hutter and J. Bechhoefer, Rev. Sci. Instrum. 64, 1868 (1993).

${ }^{15}$ M. A. Unger, S. D. O'Connor, and J. D. Baldeschwieler (unpublished). 